\title{
Holographic Storage of Biphoton Entanglement
}

\author{
Han-Ning Dai*, ${ }^{1}$ Han Zhang*, ${ }^{1}$ Sheng-Jun Yang, ${ }_{1}^{1}$ Tian-Ming Zhao, ${ }^{1}$ Jun Rui, ${ }^{1}$ You-Jin Deng, ${ }^{1}$ Li \\ $\mathrm{Li},{ }^{1}$ Nai-Le Liu, ${ }^{1}$ Shuai Chen, ${ }^{1}$ Xiao-Hui Bao, ${ }^{2}, 1$ Xian-Min Jin, ${ }^{1}$ Bo Zhao, ${ }^{1,3}$ and Jian-Wei Pan ${ }^{1}$ \\ ${ }^{1}$ Hefei National Laboratory for Physical Sciences at Microscale and Departmentof Modern Physics, \\ University of Science and Technology of China,Hefei,Anhui 230026, China \\ ${ }^{2}$ Physikalisches Institut, Reprecht-Karls-Universitat Heidelberg, Philosophenweg 12, 69120 Heidelberg, Germany \\ ${ }^{3}$ Institute for Theoretical Physics, University of Innsbruck, A-6020 Innsbruck, Austria
}

(Dated: April 4, 2022)

\begin{abstract}
Coherent and reversible storage of multi-photon entanglement with a multimode quantum memory is essential for scalable all-optical quantum information processing. Although single photon has been successfully stored in different quantum systems, storage of multi-photon entanglement remains challenging because of the critical requirement for coherent control of photonic entanglement source, multimode quantum memory, and quantum interface between them. Here we demonstrate a coherent and reversible storage of biphoton Bell-type entanglement with a holographic multimode atomicensemble-based quantum memory. The retrieved biphoton entanglement violates Bell's inequality for $1 \mu$ s storage time and a memory-process fidelity of $98 \%$ is demonstrated by quantum state tomography.
\end{abstract}

PACS numbers: 03.67.Bg, 42.50.Ex

Faithfully mapping multi-photon entanglement into and out of quantum memory is of crucial importance for scalable linear-optical quantum computation [1] and long-distance quantum communication [2]. Recently, storage of nonclassical light [3, 4] and single photons [512. has been demonstrated in various quantum systems, such as atomic ensemble, solid system, and single atom. Among these, the atomic-ensemble-based quantum memory holds the promise to implement multimode quantum memory for multi-photon entanglement. A natural method for this purpose is to select spatially separated sub-ensembles of a large atomic ensemble as different quantum registers [8, 13], for which the number of stored modes is limited by the spatial dimension of the atomic ensemble. More powerful methods, such as exploring large optical depth of an atomic ensemble [14, 15], or utilizing photon echoes [16] or atomic frequency combs techniques [17, have been employed to demonstrate atomicensemble-based multi-mode memories.

An alternative and elegant method is to implement the atomic ensemble as a holographic multimode quantum memory [18 20, using spatially overlapped but orthogonal spin waves as different quantum registers. For clarity, we illustrate this in the example of storing a single-photon state in an atomic ensemble of $N$ atoms that have two long-lived ground states $|g\rangle$ and $|s\rangle$ 21 24. Initially, a "vacuum" state $\mid$ vac $\rangle=\left|g_{1} \ldots g_{N}\right\rangle$ is prepared such that all the atoms are at the $|g\rangle$ state. The single-photon state is then mapped into the ensemble as a collective state $|1, \mathbf{q}\rangle=S_{\mathbf{q}}^{\dagger}|\mathrm{vac}\rangle=$ $(1 / \sqrt{N}) \sum_{j} e^{i \mathbf{q} \cdot \mathbf{x}_{j}}\left|g_{1} \cdots s_{j} \cdots g_{N}\right\rangle$, where $\mathbf{x}_{j}$ is the po-

[*] These authors contributed equally to this work.
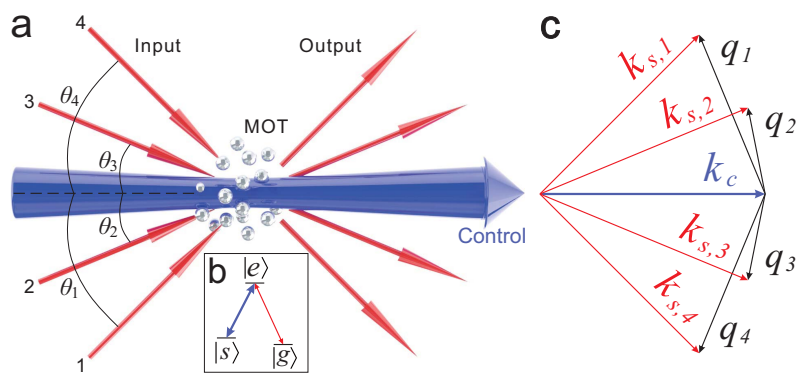

FIG. 1: (a) Schematic view of the quad-mode holographic quantum memory. The control field is shined into the atomic cloud horizontally, and the signal modes are incident from different directions in the same plane, with angles $\left(\theta_{1}, \theta_{2}, \theta_{3}, \theta_{4}\right)=\left(-1^{\circ},-0.6^{\circ}, 0.6^{\circ}, 1^{\circ}\right)$ relative to the the control field. (b) The typical $\Lambda$-type energy levels, with two ground states $|g\rangle$ and $|s\rangle$ and an excited state $|e\rangle$. The $|e\rangle-|g\rangle$ and the $|e\rangle-|s\rangle$ transition are coupled to the signal and control fields, respectively. (c) Illustration of the wave vectors of the spin waves. The input signal field with wave vector of $\mathbf{k}_{s, i}(i=1,2,3,4)$ is mapped to a spin wave with wave vector $\mathbf{q}_{i}=\mathbf{k}_{s, i}-\mathbf{k}_{c}$, with $\mathbf{k}_{c}$ the wave vector of the control field.

sition of the $j$ th atom, $S_{\mathbf{q}}^{\dagger}=(1 / \sqrt{N}) \sum_{j} e^{i \mathbf{q} \cdot \mathbf{x}_{j}}|s\rangle_{j}\langle g|$ is the collective creation operator of a spin wave with wave vector $\mathbf{q}$. For $N \gg 1$, one has $\left[S_{\mathbf{q}_{1}}, S_{\mathbf{q}_{2}}^{\dagger}\right] \approx \delta_{\mathbf{q}_{1} \mathbf{q}_{2}}$, namely, the collective states satisfy the orthogonality relation $\left\langle 1, \mathbf{q}_{1} \mid 1, \mathbf{q}_{2}\right\rangle \approx \delta_{\mathbf{q}_{1} \mathbf{q}_{2}}$. Therefore, one can encode different qubits by different phase patterns and employ a single atomic ensemble as a holographic multimode quantum memory. Since the information is stored globally throughout the medium, one can achieve high-capacity data storage. Recently, holographic storage of classical light and microwave pulses have been demonstrated [2527.

Here we report an experimental demonstration of holo- 


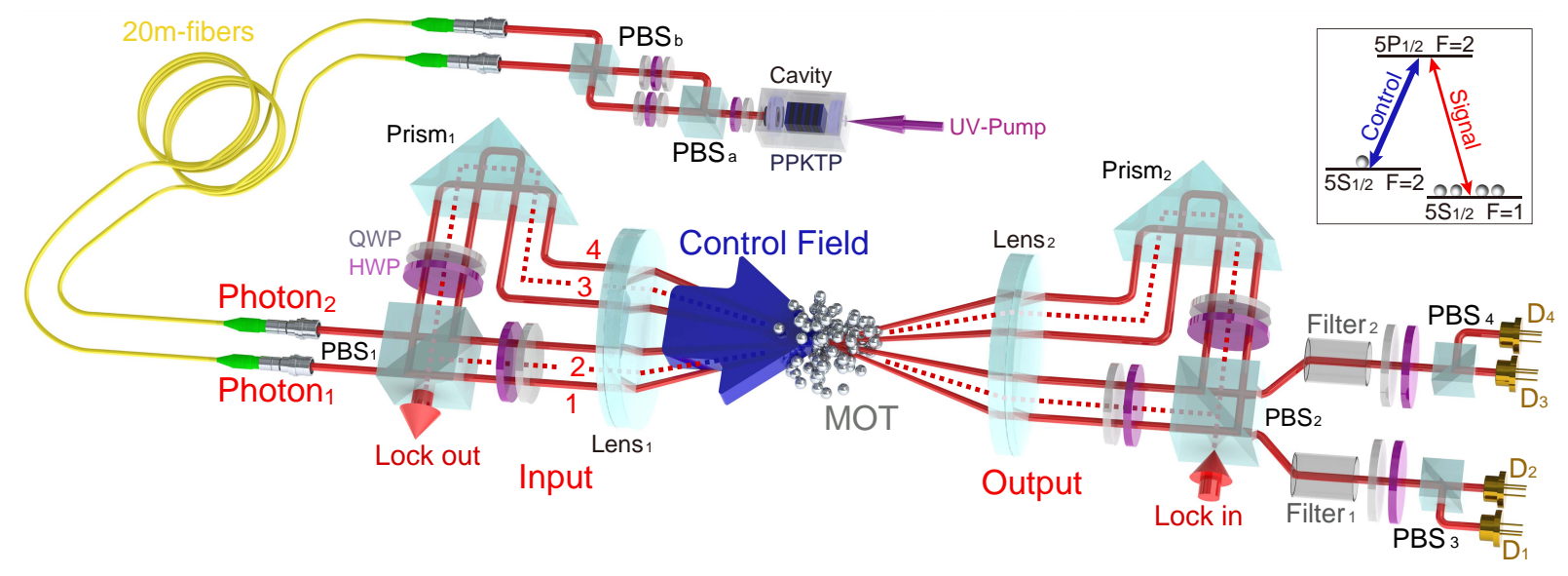

FIG. 2: Illustration of the experimental setup. The two polarization-entangled photons produced by a narrowband SPDC source are directed to the memory lab through 20-m fibers. Different polarization components are separated by the polarization beam splitter (PBS) 1, and are coupled to the quad-mode holographic quantum memory. All the light fields are turned into righthand circular polarization $\left(\sigma^{+}\right)$by wave plates. The biphoton entanglement are stored into the four quantum registers by adiabatically switching off the control light. After a controllable delay, the photons are retrieved out, transferred back to their original polarization states by wave plates and combined on $\mathrm{PBS}_{2}$. Then the retrieved entangled photons are guided into filters, containing a Fabry-Perot cavity and a hot atomic cell to filter out the leakages from the control light, and detected by single photon detectors. The path length difference between $\mathrm{PBS}_{1}$ and $\mathrm{PBS}_{2}$ is actively stabilized by an additional phase beam (dashed line). Inset: The experimental laser lights and atomic levels.

graphic storage of biphoton Bell-type entanglement with a single atomic ensemble, in which four orthogonal spin waves with different wave vectors are used as a quadmode quantum memory. The posterior biphoton entanglement is mapped into and out of the quad-register holographic quantum memory, via a technique based on electromagnetically induced transparency (EIT). Violation of Bell's inequality is observed for storage time up to 1 $\mu$ s and a memory-process fidelity of $98 \%$, calculated by quantum state tomography, is achieved.

The experimental scheme and setup are shown in Fig.1 and 2, respectively. In the memory lab, we prepare, within $14 \mathrm{~ms}$, a cold ${ }^{87} \mathrm{Rb}$ atomic ensemble consisting of about $10^{8}$ atoms in a dark Magnetic-Optical-Trap (MOT). The temperature of the atomic cloud is about $140 \mu \mathrm{K}$, and the optical depth (OD) is about 10. The typical $\Lambda$-type energy-level configuration is shown in Fig. 1 1 b, where $|g\rangle,|e\rangle$, and $|s\rangle$ correspond to the ${ }^{87} \mathrm{Rb}$ hyper-fine states $\left|5 S_{1 / 2}, F=1\right\rangle,\left|5 P_{1 / 2}, F=2\right\rangle$, and $\left|5 S_{1 / 2}, F=2\right\rangle$, respectively. All the atoms are initially prepared at $|g\rangle$.

A strong classical control field couples $|e\rangle-|s\rangle$ transition with wave vector $\mathbf{k}_{c}$ and beam waist diameter $w_{c} \approx 850$ $\mu \mathrm{m}$, while the to-be-stored quantum field, which has four components (see below), couples $|g\rangle-|e\rangle$ transition with beam waist diameter $w_{s} \approx 450 \mu \mathrm{m}$. The control field is focused at the ensemble center, and the four components of the signal field are guided through the atomic cloud along four different directions, which are in the same plane but with different angles $\theta_{i}(i=1-4)$ relative to the control-light direction [28]. We set $\left(\theta_{1}, \theta_{2}, \theta_{3}, \theta_{4}\right)=$ $\left(-1^{\circ},-0.6^{\circ}, 0.6^{\circ}, 1^{\circ}\right)$, as illustrated in Fig. 11. By care- fully adjusting the directions of the control and signal beams, we make all the light modes overlap in the center of the atomic ensemble. The atomic ensemble has a length $L \approx 2 \mathrm{~mm}$, and the signal fields propagate within the control field during storage.

Each component $i$ of the signal field is associated with a wave vector $\mathbf{k}_{s, i}$, and is to be stored in a spin wave with $\mathbf{q}_{i}=\mathbf{k}_{s, i}-\mathbf{k}_{c}$. By careful alignment, a holographic quad-mode quantum memory, with approximately equivalent optical depth and similar performance, is established. We measure the EIT transmission spectrum and perform slow-light experiment. For a control light with a Rabi frequency of about $7 \mathrm{MHz}$, we observe an EIT window of $2.2 \mathrm{MHz}$ and a delay time of about $160 \mathrm{~ns}$ for all the four modes. Note that such a holographic quantum memory is different from the scheme in Ref. 8, 13, where each signal mode requests a spatially separated atomic sub-ensemble.

The biphoton entanglement comes from a narrowband cavity-enhanced spontaneous parametric down conversion (SPDC) entanglement source as in previous work [9, 29]. The source cavity contains three main parts, i.e., a nonlinear crystal, a tuning crystal and an output coupler. The nonlinear crystal is a $25-\mathrm{mm}$ type-II a periodically poled $\mathrm{KTiOPO}_{4}$ (PPKTP) crystal, whose operational wavelength $\lambda \approx 795 \mathrm{~nm}$ is designed to match the D1 transition line of ${ }^{87} \mathrm{Rb}$. The cavity is locked intermittently to a Ti: Sapphire laser using the Pound-DreverHall method. The linewidth and finesse of the cavity are measured to be $5 \mathrm{MHz}$ and 170 , respectively.

Polarization-perpendicular photon pairs are created by 


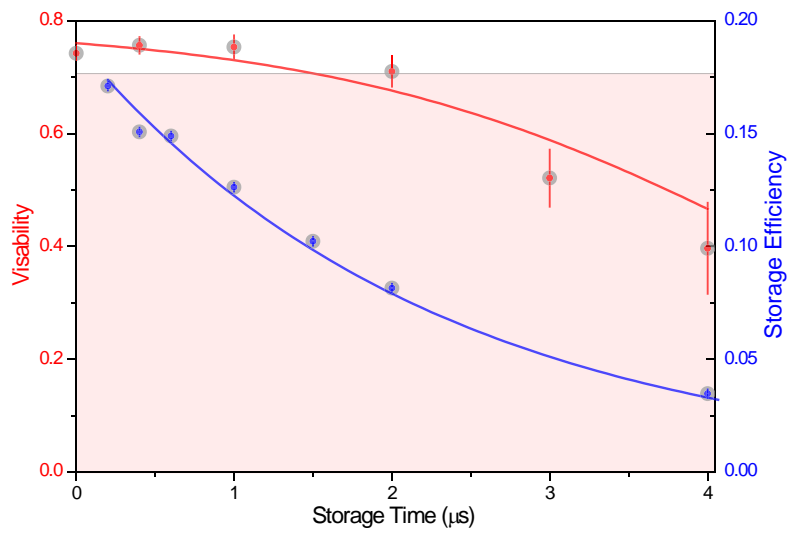

FIG. 3: Average visibility (red, left axis) and overall storage efficiency (blue, right axis) of the retrieved biphoton state versus storage time. An exponential fitting (blue solid line) of the storage efficiency yields a lifetime of $\tau=2.8 \pm 0.2$ $\mu \mathrm{s}$. The retrieved biphoton state is measured under $|H / V\rangle$, $| \pm\rangle$, and $|R / L\rangle$ bases with $| \pm\rangle=(|H\rangle \pm|V\rangle) / \sqrt{2}$, and $|R / L\rangle=(|H\rangle \pm i|V\rangle) / \sqrt{2}$. The average visibility is fitted using $V=1 /\left(a+b e^{2 t / \tau}\right)$ (red solid line) with $a$ and $b$ the fitting parameters. The result shows that within about 1.6 $\mu$ s the visibility is above the threshold of 0.71 to violate the CHSH-Bell's inequality. Error bars represent \pm standard deviation

applying a ultraviolet (UV) pumping light, which is up converted from the Ti: Sapphire laser. Single-mode output is achieved by using a filter cavity (made of a single piece of fused silica of about $6.35 \mathrm{~mm}$ ) with a finesse of 30 , which removes the background modes. Polarizationentangled photon pairs are post-selected by interfering the twin photons at polarization beam splitters (PBSs). The ideal outcome state corresponds to a Bell state

$$
\left|\phi^{+}\right\rangle_{p}=\left(|H\rangle_{1}|H\rangle_{2}+|V\rangle_{1}|V\rangle_{2}\right) / \sqrt{2}
$$

with $\mathrm{H}(\mathrm{V})$ represents the horizontal(vertical) polarization of the photons. Under a continuous wave $(\mathrm{CW})$ pump with a pump power of $4 \mathrm{~mW}$, the spectrum brightness of the polarization-entangled pairs after the filter cavity is about $50 \mathrm{~s}^{-1} \mathrm{~mW}^{-1} \mathrm{MHz}^{-1}$. In the storage experiment, the entangled signal photons are created by a $200 \mathrm{~ns}$ pump pulse, which is cut from a $28 \mathrm{~mW}$ CW pump laser. The production rate is about $33 \mathrm{~s}^{-1}$. The measured ratio of counts under $|H H\rangle /|H V\rangle$ and $|++\rangle /|+-\rangle$ bases are 14.3:1 and 23.1:1, respectively, with $| \pm\rangle=(|H\rangle \pm|V\rangle) / \sqrt{2}$.

The signal photon pair is directed to the memory lab with 20-meter single-mode fibers. The different polarization components are spatially separated by $\mathrm{PBS}_{1}$, then transferred to right-hand circular polarized $\left(\sigma^{+}\right)$by wave plates, and then guided to the four quantum registers by lens (see Fig. 22. More precisely, the $|H\rangle_{1},|H\rangle_{2},|V\rangle_{2}$, and $|V\rangle_{1}$ polarization components are coupled to mode 1-4, respectively. After these components entering the atomic ensemble, we adiabatically switch off the control light, and the photonic entanglement is mapped into the atomic ensemble. This yields an entanglement among the four quantum registers

$$
|\psi\rangle_{a}=\left(S_{\mathbf{q}_{1}}^{\dagger} S_{\mathbf{q}_{2}}^{\dagger}+S_{\mathbf{q}_{3}}^{\dagger} S_{\mathbf{q}_{4}}^{\dagger}\right)|\mathrm{vac}\rangle / \sqrt{2}
$$

After a controllable delay, we adiabatically switch on the control light and convert the atomic entanglement back into photonic entanglement. The polarization states of the output photons are transferred back linearly polarized by wave plates and combined by $\mathrm{PBS}_{2}$ to reconstruct the biphoton entanglement. The two retrieved entangled photons are respectively guided into a filter consisting of a Fabry-Perot cavity (transmission window 600 $\mathrm{MHz}$ ) and a pure ${ }^{87} \mathrm{Rb}$ vapor cell with atoms prepared in $\left|5 S_{1 / 2}, F=2\right\rangle$, and then detected by single-photon detectors. The measured overall average storage efficiency is shown in Fig. 3, which yields a 1/e lifetime of $2.8 \pm 0.2$ $\mu \mathrm{s}$. The measured coincidence rate without storage and after $1 \mu$ s storage time is $1.3 \mathrm{~s}^{-1}$ and $0.03 \mathrm{~s}^{-1}$, respectively. The propagating phase between $\mathrm{PBS}_{1}$ and $\mathrm{PBS}_{2}$ is actively stabilized within $\lambda_{l} / 30$ by an additional phase lock beam with $\lambda_{l} \approx 780 \mathrm{~nm}[9,28$.

To verify that the biphoton Bell-type entanglement is faithfully mapped into and out of the four holographic quantum registers, we first measure the retrieved biphoton state in $|H / V\rangle,| \pm\rangle$, and $|R / L\rangle=(|H\rangle \pm i|V\rangle) / \sqrt{2}$ bases at different storage time. The average visibility is shown in Fig. 3, which for storage time less than $1.6 \mu \mathrm{s}$, exceeds the threshold 0.71 to violate CHSHBell's inequality. Note that the reduction of the visibility with storage time is mainly due to the background coincidences caused by the dark counts and the leakage from the control field. We further measure the correlation function $E\left(\phi_{1}, \phi_{2}\right)$, with $\phi_{1}\left(\phi_{2}\right)$ the polarization angle for signal photon 1(2), and calculate quantity $S=\left|-E\left(\phi_{1}, \phi_{2}\right)+E\left(\phi_{1}, \phi_{2}^{\prime}\right)+E\left(\phi_{1}^{\prime}, \phi_{2}\right)+E\left(\phi_{1}^{\prime}, \phi_{2}^{\prime}\right)\right|$, where $\left(\phi_{1}, \phi_{1}^{\prime}, \phi_{2}, \phi_{2}^{\prime}\right)=\left(0^{\circ}, 45^{\circ}, 22.5^{\circ}, 67.5^{\circ}\right)$. We obtain $S=2.54 \pm 0.03$ for the input state, and $S=2.25 \pm 0.08$ for the retrieved state after $1 \mu \mathrm{s}$ storage. The violation of the CHSH-Bell's inequality $(S>2)$ 30 confirms the entanglement has been coherently and reversibly stored in the quad-mode holographic quantum memory.

To quantitatively assess the fidelity of the storage process, we perform the quantum state tomography [31, 32. to construct the density matrix $\rho_{\text {in }}$ of the input and $\rho_{\text {out }}$ of the output state after $1 \mu$ s storage, in which the polarization state of each photon is measured with two singlephoton detectors under different detection settings. The results are illustrated in Fig. 4, from which the fidelity of the measured state $\rho$ on the ideal Bell state $\rho_{\phi^{+}}$is calculated as $F\left(\rho_{\phi^{+}}, \rho\right)=\left(\operatorname{Tr}\left(\sqrt{\sqrt{\rho_{\phi^{+}}} \rho \sqrt{\rho_{\phi^{+}}}}\right)\right)^{2}$. A Monte Carlo simulation technique 32 is applied to calculate the uncertainties of the fidelity. Briefly, an ensemble of 100 random sets of data are generated according to Possionian distribution and then the density matrices are 

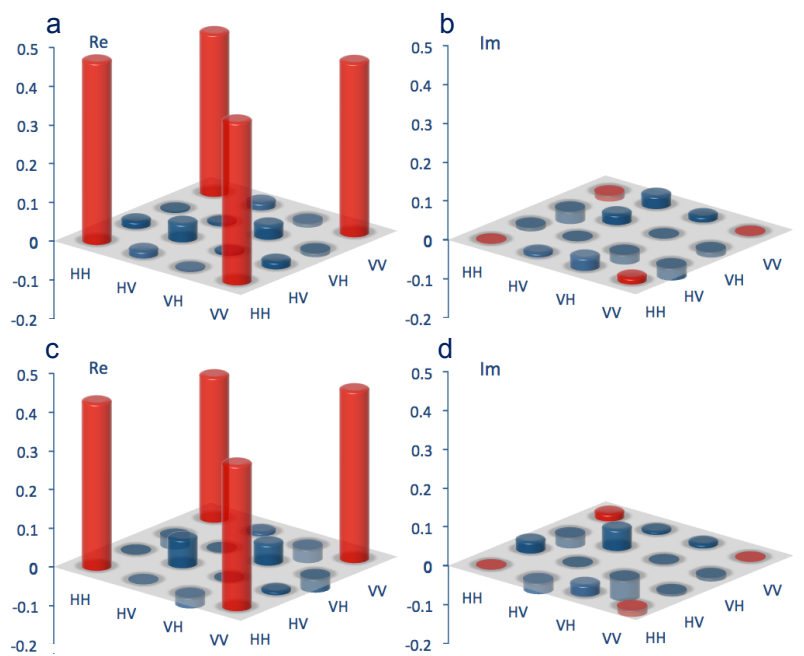

FIG. 4: Density matrix of the input state $(\mathbf{a}, \mathbf{b})$ and of the output state after $1 \mu$ s storage $(\mathbf{c}, \mathbf{d})$, obtained from quantum state tomography. $\mathbf{a}$ and $\mathbf{c}$ are for the real parts, and $\mathbf{b}$ and d are for the imaginary parts.

obtained by means of the maximum likelihood method. This yields a distribution of fidelities, from which the mean value and uncertainties of the fidelity are calculated. We obtain $F\left(\rho_{\phi^{+}}, \rho_{\text {in }}\right)=(87.9 \pm 0.5) \%$ for the input state $\rho_{\text {in }}$ and $F\left(\rho_{\phi^{+}}, \rho_{\text {out }}\right)=(81 \pm 2) \%$, beyond the threshold [33] $78 \%$ for Werner states to violate Bell's inequality. The fidelity of the memory process is given by $F\left(\rho_{\text {in }}, \rho_{\text {out }}\right)=(98.2 \pm 0.9) \%$.

In summary, we have experimentally demonstrated the coherent mapping of a biphoton Bell-type entanglement, created from a narrowband SPDC source, into and out of a four-register holographic quantum memory, with a high memory-process fidelity of $98 \%$ for $1 \mu$ s storage time. The narrowband photonic entanglement source inherits the advantage of conventional broadband SPDC source, and can be used to generate multi-photon entanglement beyond biphoton entanglement. A novel feature of the holographic quantum memory is that one can use more modes by simply choosing the directions of the signal and control fields. The memory capacity $N_{m}$ for a coplanar configuration may be estimated by the geometric mean of the Fresnel numbers of the illuminated regions as $N_{m} \sim w_{c} w_{s} /(\lambda L)$ [20, which is $N_{m} \sim 240$ for our experimental parameters. Increasing the beam waist diameters or extending to a three-dimensional geometry would allow much more modes. Individual control of each quantum register may be achieved by using an optical cavity and employing the stimulated Raman adiabatic passage technique [18] or employing the phase match method [20].

To extend our work to storage of multi-photon entanglement, we have to improve the brightness of the entanglement source, and increase the retrieval efficiency and lifetime of the quantum memory. The storage efficiency is about $15 \%$, which can be improved by further increasing the optical depth and reducing the linewidth of the narrowband entanglement source. The storage time is about $1 \mu \mathrm{s}$, which is limited by inhomogeneous broadening induced by residual magnetic field, and can be improved to be of order of millisecond by trapping the atoms in optical lattice and using the magnetic-insensitive state [34, 35]. Our work opens up the possibility of scalable preparation and high-capacity storage of multi-photon entanglement, and also sheds light on the emerging field of holographic quantum information processing.

This work was supported by the National Natural Science Foundation of China, the National Fundamental Research Program of China (grant no. 2011CB921300), the Chinese Academy of Sciences, the Austrian Science Fund, the European Commission through the European Research Council Grant and the Specific Targeted Research Projects of Hybrid Information Processing.

[1] E. Knill, R. LafLamme, and G.J. Milburn, Nature 409, 46 (2001)

[2] L.-M. Duan, M.D. Lukin, J.I. Cirac, and P. Zoller, Nature 414, 413 (2001).

[3] B. Julsgaard, et al., Nature 432, 482 (2004).

[4] J. Appel, E. Figueroa, D. Korystov, M. Lobino, and A.I. Lvovsky, Phys. Rev. Lett. 100, 093602 (2008).

[5] T. Chanelière, et al., Nature 438, 833 (2005).

[6] M.D. Eisaman, et al., Nature 438, 837 (2005).

[7] K.S. Choi, H. Deng, J. Laurat, and H.J. Kimble, Nature 452, 67 (2008).

[8] K.S. Choi, et al., Nature 468, 412 (2010).

[9] H. Zhang, et al., Nat. Photon. 5, 628 (2011).

[10] C. Clausen, et al., Nature 469, 508 (2011).

[11] E. Saglamyurek, et al., Nature 469, 512 (2011).

[12] H.P. Specht, Nature 473, 190 (2011).

[13] S.-Y. Lan, et al., Opt. Express 17, 13639 (2009).

[14] J. Nunn, et al., Phys. Rev. Lett. 101, 260502 (2008).

[15] E. Zeuthen, A. Grodecka-Grad, and A.S. Sørensen, Phys. Rev. A 84, 043838 (2011).

[16] M. Hosseini, et al., Nature 461, 241 (2009).

[17] H. de Riedmatten, et al., Nature 456, 773 (2008).

[18] K. Tordrup, A. Negretti, and K. Mølmer, Phys. Rev. Lett. 101, 040501 (2008).

[19] D.V. Vasilyev, I.V. Sokolov, and E.S. Polzik, Phys. Rev. A 77, 020302 (2008).

[20] K. Surmacz, et al., Phys. Rev. A 78, 033806 (2008).

[21] M. Fleischhauer and M.D. Lukin, Phys. Rev. Lett. 84, 5094 (2000).

[22] C. Liu, Z. Dutton, C.H. Behroozi, and L.V. Hau, Nature 409, 490 (2001).

[23] D.F. Phillips, A. Fleischhauer, A. Mair, R.L. Walsworth, and M.D. Lukin, Phys. Rev. Lett. 86, 783 (2001).

[24] N.B. Phillips, A.V. Gorshkov, and I. Novikova, Phys. Rev. A 78, 023801 (2008).

[25] P.K. Vudyasetu, R.M. Camacho, and J.C. Howell, Phys. Rev. Lett. 100, 123903 (2008).

[26] M. Shuker, O. Firstenberg, R. Pugatch, A. Ron, and N. Davidson, Phys. Rev. Lett. 100, 223601 (2008).

[27] H. Wu, et al., Phys. Rev. Lett. 105, 140503 (2010). 
[28] S. Chen, et al., Phys. Rev. Lett. 99, 180505 (2007).

[29] X.-H. Bao, et al., Phys. Rev. Lett. 101, 190501 (2008).

[30] J.F. Clauser, M.A. Horne, A. Shimony, and R.A. Holt, Phys. Rev. Lett. 23, 880 (1969).

[31] G.M. D'Ariano, M.G.A. Paris, M.F. Sacchi, Adv. Imag. Elec. Phys. 128, 205 (2003).
[32] J.B. Altepeter, E.R. Jeffrey, and P.G. Kwiat, Adv. At. Mol. Opt. Phys. 52, 105 (2005).

[33] M. Aspelmeyer, et al., Science 301, 621 (2003).

[34] B. Zhao, et al., Nat. Phys. 5, 95 (2009).

[35] R. Zhao, et al., Nat. Phys. 5, 100 (2009). 